\title{
CONTINUOUS-TRACE ALGEBRAS FROM THE BUNDLE THEORETIC POINT OF VIEW
}

\author{
JONATHAN ROSENBERG
}

(Received 14 January 1988)

Communicated by W. Moran

\begin{abstract}
Using various facts about principal bundles over a space, we give a unified treatment of several theorems about the structure of stable separable continuous-trace algebras, their automorphisms, and their $K$-theory. We also present a classification of real continuous-trace algebras from the same point of view.
\end{abstract}

1980 Mathematics subject classification (Amer. Math. Soc.) (1985 Revision): 46 L 05, 46 L 35, 46 L 80, 46 M 20, 55 R 10, 19 K 99.

This paper collects together a number of observations about continuous-trace $C^{*}$-algebras, especially in the stable separable case. While I do not claim any great originality for any of these remarks, I have tried to do everything from the uniform point of view of the topology of bundles, which I have found helpful over the years. Thus I hope these comments will be of some value to others. I would like to thank the Centre for Mathematical Analysis, and in particular the organisers of the August 1987 miniconference on operator algebras and harmonic analysis, for helping to make this project possible. Parts of Section 3 below were presented at the conference on operator algebras in Santa Barbara, California, in 1986.

\footnotetext{
This research was partially supported by the National Science Foundation of the U.S.A., Grant no. DMS-87-00551. The paper was written while the author was visiting the Centre for Mathematical Analysis at the Australian National University, Canberra, July-August, 1987.

(C) 1989 Australian Mathematical Society 0263-6115/89 \$A2.00+0.00
} 


\section{Complex stable continuous-trace algebras and their automorphisms}

Recall that a $C^{*}$-algebra $A$ (for the moment we are working over $\mathbb{C}$-we will consider the real case separately, later) is said to have continuous trace if $\hat{A}$ is Hausdorff and if the continuous-trace elements

$\left\{a \in A_{+} \mid \operatorname{Tr} \pi(a)<\infty\right.$ for all $\pi \in \hat{A}$, and $\pi \mapsto \operatorname{Tr} \pi(a)$ is continuous on $\left.\hat{A}\right\}$

are dense in $A_{+}$. Fix once and for all a separable, infinite-dimensional Hilbert space $\mathscr{H}$ and let $\mathscr{B}(\mathscr{H})$ and $\mathscr{K}=\mathscr{K}(\mathscr{H})$ denote the algebras of bounded and compact operators on $\mathscr{H}$, respectively. We shall need to rely constantly on the following basic results of Dixmier and Douady ([5]—see also [4] for a good exposition), which for simplicity we state only in the separable case, though they basically also apply when $\hat{A}$ is paracompact.

THEOREM 1.1 (Dixmier and Douady [5]). Let $A$ be a separable continuoustrace algebra with spectrum $X$. Then $A \cong \Gamma_{0}(\mathscr{A})$, the algebra of sections vanishing at infinity of a continuous field $\mathscr{A}$ of elementary $C^{*}$-algebras over $X$. To $\mathscr{A}$ is associated a characteristic class $\delta(A) \in H^{3}(X, \mathbf{Z})$ (Cech cohomology). If $A$ is stable, that is $A \cong A \otimes \mathscr{K}$, then $\mathscr{A}$ is locally trivial, with fibres $\cong \mathscr{K}$. In this case, $A$ is determined, up to automorphisms fixing $X$ pointwise, by $\delta(A)$. And any class $\delta \in H^{3}(X, \mathbf{Z})$ arises from a (unique) stable separable continuous-trace algebra $A_{\delta}$ over $X$.

This theorem makes possible the understanding of stable separable continuous-trace algebras from the point of view of bundle theory. To fix notation, let

$U(D)=$ the unitary group of a $C^{*}$-algebra $D$, with the norm topology, $U=\{u \in U(\mathscr{B}(\mathscr{H})) \mid u-1 \in \mathscr{K}\}$,

$\mathscr{U}=U(\mathscr{B}(\mathscr{H}))$, but with the weak operator topology,

$P \mathscr{U}=\mathscr{U} / \mathbf{T}$, which may be identified with $\operatorname{Aut}(\mathscr{K})$, the group of

*automorphisms of $\mathscr{K}$ with the topology of pointwise

convergence, via the isomorphism $\dot{u} \mapsto \mathrm{Ad} u$,

$\mathscr{Q}=\mathscr{B}(\mathscr{H}) / \mathscr{K}(\mathscr{H})$, the Calkin algebra.

It is well known that $U, \mathscr{U}, P \mathscr{U}, U(\mathscr{B}(\mathscr{H}))$, and $U(\mathscr{Q})$ all have the homotopy types of countable CW-complexes. In fact, $\mathscr{U}$ [5, Section 11, Lemma 3] and $U(\mathscr{B}(\mathscr{H}))$ [9] are contractible, $P \mathscr{U}$ is a $K(\mathbf{Z}, 2)$ space (since it is the base space of a locally trivial principal $\mathbf{T}$-bundle with contractible total space) and $U$ and $U(\mathscr{Q})$ are classifying spaces for $K^{-1}$ and $K^{0}$, complex $K$-theory (see for instance [8, III.7.10]; these facts go back to Atiyah and Jänich). Also note that via the identification of $P \mathscr{U}$ with Aut $\mathscr{K}, P \mathscr{U}$ acts on $U$ and on $U(\mathscr{Q})$. 
Now fix a second-countable locally compact space $X$ and let $\delta \in H^{3}(X, Z)$. We may view $\delta$ as a homotopy class of maps $X \rightarrow K(Z, 3) \simeq B P \mathscr{U}$, or as defining a principal $P \mathscr{U}$-bundle

$$
\begin{gathered}
P \mathscr{U} \rightarrow Y=Y_{\delta} \\
\downarrow p \\
X .
\end{gathered}
$$

(It will be easiest to let $P \mathscr{U}$ act on $Y$ on the right.) Then we may form the associated bundle $\mathscr{A}_{\delta}=Y_{\delta} \times_{P \mathscr{U}} \mathscr{K}$, where $P \mathscr{U}$ acts on $\mathscr{K}$ by ${ }^{*}$-automorphisms, and we have $A_{\delta}=\Gamma_{0}\left(X, \mathscr{A}_{\delta}\right)$. Thus knowledge of the $C^{*}$-algebra $A_{\delta}$ is equivalent to understanding of the principal $P \mathscr{U}$-bundle $p: Y_{\delta} \rightarrow X$.

Now we may use this picture to describe the automorphisms of $A_{\delta}$ and to recover some results of Phillips and Raeburn [13]. It is clear that the automorphisms of $A_{\delta}$ correspond exactly to bundle automorphisms of $\mathscr{A}_{\delta} \rightarrow$ $X$, and thus, since $P \mathscr{U}$ is the full *-automorphism group of the fibres of $\mathscr{A} \delta$, to the $P \mathscr{U}$-equivariant self-homeomorphisms of $Y_{\delta}$.

Next, introduce sheaves $\mathscr{E}_{\delta}$ and $\mathscr{F}_{\delta}$ over $X$ by taking germs of $P \mathscr{U}$-equivariant maps on $Y_{\delta}$. More precisely, let $\mathscr{U}_{\text {Ad }}$ and $P \mathscr{U}_{\text {Ad }}$ denote $\mathscr{U}$ and $P \mathscr{U}$, viewed as $P \mathscr{U}$-spaces via the right adjoint action:

$$
u \cdot \dot{v}=v^{-1} u v, \quad u \in \mathscr{U}, \dot{v} \in P \mathscr{U} \text { the image of } v \in \mathscr{U} .
$$

For an open set $V$ in $X$, we let

$$
\begin{aligned}
& \Gamma\left(V, \mathscr{E}_{\delta}\right)=\left\{P \mathscr{U} \text {-equivariant continuous maps } p^{-1}(V) \rightarrow \mathscr{U}_{\mathrm{Ad}}\right\}, \\
& \Gamma\left(V, \mathscr{F}_{\delta}\right)=\left\{P \mathscr{U} \text {-equivariant continuous maps } p^{-1}(V) \rightarrow P \mathscr{U}_{\mathrm{Ad}}\right\},
\end{aligned}
$$

where $p: Y_{\delta} \rightarrow X$ is the bundle projection. It is easy to see that $\mathscr{E}_{\delta}$ and $\mathscr{F}_{\delta}$ define sheaves over $X$, and in fact, they are locally isomorphic to $\underline{\mathscr{U}}$ and $P \mathscr{U}$, respectively, the sheaves of germs of continuous functions on $X$ with values in $\mathscr{U}$ and $P \mathscr{U}$. For if $V$ is sufficiently small, $p^{-1}(V) \cong V \times P \mathscr{U}$, and a $P \mathscr{U}$-equivariant map on $p^{-1}(V)$ is determined uniquely by its values on $V \times\{1\}$.

Let Aut $A_{\delta}$ denote the group of automorphisms of $A_{\delta}$ which fix the spectrum $X$ pointwise, and let Inn $A_{\delta}$ denote the group of inner automorphisms ( $=\left\{\operatorname{Ad} u \mid u \in U\left(M\left(A_{\delta}\right)\right)\right\}$ ). Let Homeo $\delta$ denote the group of homeomorphisms $\phi: X \rightarrow X$ with $\phi^{*} \delta=\delta$.

THEOREM 1.2 (Phillips and Raeburn [13]). There are short exact sequences of groups

(a) $1 \rightarrow$ Aut $_{X} A_{\delta} \rightarrow$ Aut $A_{\delta} \rightarrow$ Homeo $_{\delta} X \rightarrow 1$,

(b) $1 \rightarrow \operatorname{Inn} A_{\delta} \rightarrow$ Aut $_{X} A_{\delta} \rightarrow H^{2}(X, \mathbb{Z}) \rightarrow 1$. 
Proof. (a) Obviously any $P \mathscr{U}$-equivariant homeomorphism of $Y_{\delta}$ sends fibres of $p$ to fibres of $p$, and thus gives rise to a homeomorphism of $X$, which fixes $\delta$ since it lifts to the bundle $Y_{\delta}$. So the only thing non-obvious is the surjectivity. But if $\phi \in \mathrm{Homeo}_{\delta} X$, then $\phi^{*} Y_{\delta}$ is isomorphic to $Y_{\delta}$ as a principal $P \mathscr{U}$-bundle. Choose a $P \mathscr{U}$-bundle isomorphism $\psi$. We have a commutative diagram

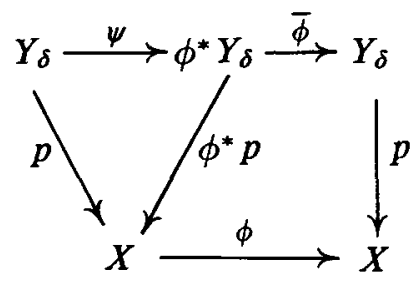

and $\bar{\phi} \circ \psi$ is a $P \mathscr{U}$-equivariant homeomorphism of $Y_{\delta}$, lifting $\phi$.

(b) The second part of the theorem will follow easily from the following.

Proposition 1.3. There is a short exact sequence of sheaves (of groups over $X)$

$$
1 \rightarrow \underline{\mathbf{I}} \rightarrow \mathscr{E}_{\delta} \rightarrow \mathscr{F}_{\delta} \rightarrow 1,
$$

where $\mathbf{I}$ is the sheaf of germs of continuous $\mathbf{T}$-valued functions. Furthermore, there is a natural isomorphism

$$
\text { Aut }_{X} A_{\delta} \cong H^{0}\left(X, \mathscr{F}_{\delta}\right) \text {, }
$$

and $\operatorname{Inn} A_{\delta}$ is identified with the image in this group of $H^{0}\left(X, \mathscr{E}_{\delta}\right)$.

Proof. The first statement is immediate from the exact sequence

$$
1 \rightarrow \mathbf{T} \rightarrow \mathscr{U} \rightarrow P \mathscr{U} \rightarrow 1
$$

and the fact that the adjoint action of $P \mathscr{U}$ on $T$ is trivial so that a $P \mathscr{U}$ equivariant map $p^{-1}(V) \rightarrow \mathrm{T}$ factors uniquely through $V$.

For the second statement, suppose $\phi: Y_{\delta} \rightarrow Y_{\delta}$ is $P \mathscr{U}$-equivariant and commutes with projection to $X$, that is, represents an element of $\mathrm{Aut}_{X} A_{\delta}$. Then we may write $\phi(y)=y \cdot g(y)$, where $g: Y \rightarrow P \mathscr{U}$, since $\phi(y)$ and $y$ lie in the same fibre of $p$ and $P \mathscr{U}$ acts simply transitively on fibres. Furthermore, given $\dot{v} \in P \mathscr{U}$, we have

$$
\begin{aligned}
\phi(y \cdot \dot{v}) & =\phi(y) \cdot \dot{v} \quad \text { (by equivariance of } \phi) \\
& =y \cdot g(y) \dot{v}=(y \cdot \dot{v}) \cdot \dot{v}^{-1} g(y) \dot{v},
\end{aligned}
$$


so $g(y \cdot \dot{v})=\dot{v}^{-1} g(y) \dot{v}$ for $y \in Y, \dot{v} \in P \mathscr{U}$, and $g$ is equivariant for the right adjoint action of $P \mathscr{U}$ on itself. Reversing the calculations, such a $g$ defines a $P \mathscr{Z}$-equivariant map $\phi: Y_{\delta} \rightarrow Y_{\delta}$ by $\phi(y)=y \cdot g(y)$. Thus

$$
\text { Aut }_{X} A_{\delta} \cong\left\{P \mathscr{U} \text {-equivariant continuous maps } Y_{\delta} \rightarrow P \mathscr{U}_{\mathrm{Ad}}\right\} \text {. }
$$

On the other hand, a unitary of the multiplier algebra of $A_{\delta}$ is represented [2, Corollary 3.5] by a section of the bundle of copies of $\mathscr{U}$ associated to $p: Y_{\delta} \rightarrow X$, that is, by a continuous section $X \rightarrow Y \times_{P \mathscr{U} \text {, Ad }} \mathscr{U}$. Here $P \mathscr{U}$ acts via the left adjoint action on $\mathscr{U}$. But given such a section $s: x=p(y) \mapsto$ $[(y, u)]$ and a choice of $y \in p^{-1}(x)$, the $u \in \mathscr{U}$ is uniquely determined by $y$, that is, may be written as $g(y)$ for $g: Y \rightarrow \mathscr{U}$ continuous, and the condition $[(y, g(y))]=[(y \cdot \dot{v}, g(y \cdot \dot{v}))]$ in the fibre product forces $g(y \cdot \dot{v})=\dot{v}^{-1} \cdot g(y)=$ $v^{-1} g(y) v$, so $g$ is equivariant for the right adjoint action of $P \mathscr{U}$. Once again, the argument is reversible, so $U\left(M\left(A_{\delta}\right)\right) \cong H^{0}\left(X, \mathscr{E}_{\delta}\right)$ (as groups; to make the isomorphism topological, one should use the strict topology on multipliers).

It is now easy to see that the action of unitary multipliers by inner automorphisms is given by the natural map $H^{0}\left(X, \mathscr{E}_{\delta}\right) \rightarrow H^{0}\left(X, \mathscr{F}_{\delta}\right)$.

Proof of 1.2 (continued). To finish the proof, we consider the cohomology exact sequence

$$
1 \rightarrow H^{0}(X, \underline{\mathbf{I}}) \rightarrow H^{0}\left(X, \mathscr{E}_{\delta}\right) \stackrel{\alpha}{\rightarrow} H^{0}\left(X, \mathscr{F}_{\delta}\right) \rightarrow H^{1}(X, \underline{\mathrm{I}}) \rightarrow H^{1}\left(X, \mathscr{E}_{\delta}\right) .
$$

By 1.3, the image of $\alpha$ may be identified with $\operatorname{Inn} A_{\delta}$ sitting inside Aut $_{X} A_{\delta}$. In fact, $H^{0}(X, \underline{\mathbf{I}})=C(X, \mathrm{~T})$ is just the centre of $U\left(M\left(A_{\delta}\right)\right) \cong H^{0}\left(X, \mathscr{E}_{\delta}\right)$ and $\alpha=$ Ad, which kills the centre. Furthermore, because of the exact sequence $1 \rightarrow \mathbf{Z} \rightarrow \underline{\mathbf{R}} \rightarrow \underline{\mathbf{I}} \rightarrow 1$ and the fact that $\underline{\mathbf{R}}$ is a fine sheaf, $H^{1}(X, \underline{\mathbf{I}}) \cong H^{2}(X, \mathbf{Z})$. Finally, recall that $\mathscr{E}_{\delta}$ is locally isomorphic to $\underline{\mathscr{L}}$ [5, Section 11 , Lemma 4], this is a soft sheaf (since $\mathscr{U}$ is contractible), and since softness is a local property, $\mathscr{E}_{\delta}$ is soft as well. Hence $H^{1}\left(X, \mathscr{E}_{\delta}\right)=1$ and the theorem follows.

\section{Twisted $K$-theory}

Given a second-countable locally compact space $X$ and $\delta \in H^{3}(X, \mathbf{Z})$, we define the corresponding twisted $K$-groups of $X$ by

$$
K^{-i}(X, \delta)=K_{i}\left(A_{\delta}\right),
$$

where $A_{\delta}$ is as defined above. When $\delta$ is a torsion class and $X$ is a finite CWcomplex, then there is a (non-unique) continuous-trace algebra $B_{\delta}$ over $X$ with Dixmier-Douady invariant $\delta$ and with all irreducible representations of $B_{\delta}$ of bounded finite degree; in fact, $B_{\delta}$ is an Azumaya algebra over $C(X)$ in the usual sense of ring theory [7]. In this case there is a spectrum-preserving 
strong Morita equivalence between $B_{\delta}$ and $A_{\delta}$, so our twisted $K$-groups agree with those defined by Donovan and Karoubi [6] - see also [12]. The twisted $K$-groups may be computed purely topologically using the following elementary proposition.

Proposition 2.1. Suppose $X$ is compact. There are natural identifications

$$
K^{0}(X, \delta) \cong\left[Y_{\delta}, U(\mathscr{Q})\right]^{P \mathscr{U}}
$$

and

$$
K^{-1}(X, \delta) \cong\left[Y_{\delta}, U\right]^{P \mathscr{U}} .
$$

Here $[,]^{P \mathscr{L}}$ denotes the space of homotopy classes of PQU-equivariant maps, that is, $\pi_{0}\left(\operatorname{Map}(,)^{P \mathscr{U}}\right)$, and $P \mathscr{U}$ acts on $U$ and on $U(\mathscr{Q})$ via its action by *automorphisms of $\mathscr{K}$.

Proof. Recall that $K_{1}\left(A_{\delta}\right)$ is the group of path-components of $\left\{u \in U\left(A_{\delta}^{+}\right) \mid\right.$ $\left.u-1 \in A_{\delta}\right\}$, where $A_{\delta}^{+}$denotes the $C^{*}$-algebra obtained from $A_{\delta}$ by adjoining an identity. (This is because $A_{\delta}$ is stable-see, for instance, [15, Theorem 4.1].) But $\left\{u \in U\left(A_{\delta}^{+}\right) \mid u-1 \in A_{\delta}\right\}$ is obviously isomorphic to the group of sections of the $U$-bundle associated to $p$, that is, to sections $X \rightarrow Y_{\delta} \times_{P \mathscr{C}} U$. As in the proof of Proposition 1.3 above, such sections may be identified with $P \mathscr{U}$-equivariant maps $Y_{\delta} \rightarrow U$. This gives the calculation of $K^{-1}(X, \delta)$. The calculation of $K_{0}\left(A_{\delta}\right)$ is similar, using the identification of $K_{0}\left(A_{\delta}\right)$ with the path components of the unitary groups of $\mathscr{Q}_{\delta}=\Gamma\left(X, Y_{\delta} \times_{P \mathscr{C}} \mathscr{Q}\right)$ (see for instance [11]).

Now we may rederive a result (Theorem 6.5) of [14]. We also obtain a new way of controlling the differentials in the spectral sequence.

Theorem 2.2. Let $X$ be a finite $\mathrm{CW}$-complex and $\delta \in H^{3}(X, \mathbf{Z})$. There is a strongly convergent spectral sequence

$$
H^{p}\left(X, K^{q}(p t)\right) \Rightarrow K^{*}(X, \delta),
$$

and in fact there is a commuting diagram of spectral sequences of Leray-Serre, Atiyah-Hirzebruch, G. Segal ([17, Proposition 5.2]) type

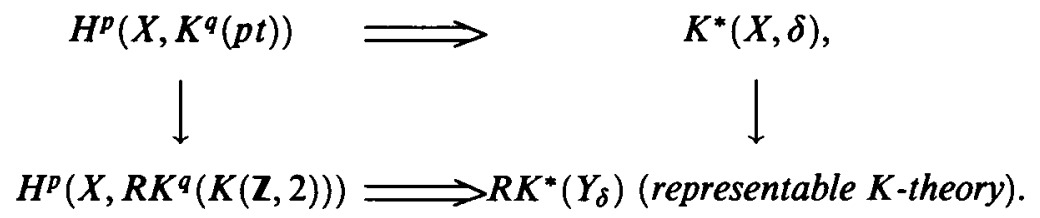

Both spectral sequences are periodic of period 2 in $q$, with $E_{2}^{p, q}=0$ for $q$ odd, and for $q$ even, $R K^{q}(K(\mathbf{Z}, 2)) \cong \mathbf{Z}[[c]]$, where the generator $c$ corresponds to the first Chern class. 
Proof. The forgetful maps $\left[Y_{\delta}, U(\mathscr{Q})\right]^{P \mathscr{U}} \rightarrow\left[Y_{\delta}, U(\mathscr{Q})\right],\left[Y_{\delta}, U\right]^{P \mathscr{U}} \rightarrow$ $\left[Y_{\delta}, U\right]$ induce natural maps $K^{*}(X, \delta) \rightarrow R K^{*}\left(Y_{\delta}\right)$. The spectral sequence compatible with this may be obtained by the method of [17, Proposition 5.2] or merely by filtering $Y_{\delta}$ according to the skeletal filtration of $X$ (cf. [1, Part III, Section 7]. Over a single cell $\mathbf{R}^{n} \subset X$, we have $p^{-1}\left(\mathbf{R}^{n}\right) \cong \mathbf{R}^{n} \times P \mathscr{U}$, so $K^{*}\left(\mathbf{R}^{n}, \delta\right) \cong K^{*}\left(\mathbf{R}^{n}\right)$. Similarly $R K^{*}\left(p^{-1}\left(D^{n}\right), p^{-1}\left(\partial D^{n}\right)\right) \cong K^{*}\left(\mathbf{R}^{n}\right) \otimes$ $R K^{0}(P \mathscr{U})$. Thus we can argue exactly as in [1, Part III, Section 7].

For the final remark, recall $P \mathscr{U} \simeq K(Z, 2) \simeq B \mathrm{~T}$, so that by the Atiyah and Segal theorem [3], $R K^{-1}(P \mathscr{U})=0$ and $R K^{0}(P \mathscr{U}) \cong R(\mathrm{~T})^{-}=\mathrm{Z}\left[t, t^{-1}\right]^{\sim}$, the completion of the Laurent polynomial ring in one variable with respect to the $I$-adic topology, where the augmentation ideal $I$ is generated by $c=t-1$. This gives the formal power series ring $\mathbf{Z}[[c]]$. (It is not necessary to invert $t=c+1$ since $t^{-1}=(1+c)^{-1}=1-c+c^{2}-\cdots$.)

The usefulness of the diagram

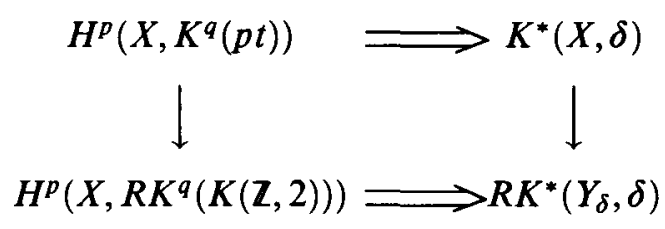

may be illustrated by the following example which was also considered in [14, Section 6], from a somewhat different point of view. Let $X=S^{3}$, let $x$ be the standard generator of $H^{3}\left(S^{3}, \mathbf{Z}\right)$, and let $\delta=N x, N \in \mathbf{Z}$. Then the homotopy sequence of the fibration $P \mathscr{U} \rightarrow Y_{\delta} \rightarrow S^{3}$ reduces to

$$
\cdots \rightarrow \pi_{n}(K(\mathbf{Z}, 2)) \rightarrow \pi_{n}\left(Y_{\delta}\right) \rightarrow \pi_{n}\left(S^{3}\right) \rightarrow \pi_{n-1}(K(\mathbf{Z}, 2)) \rightarrow \cdots,
$$

so $Y_{\delta}$ is 1 -connected and we have an exact sequence

$$
0 \rightarrow \pi_{3}\left(Y_{\delta}\right) \rightarrow \mathbf{Z} \stackrel{N}{\rightarrow} \mathbf{Z} \rightarrow \pi_{2}\left(Y_{\delta}\right) \rightarrow 0 .
$$

Thus if $y$ is the standard generator of $H^{2}(K(\mathbf{Z}, 2), \mathbf{Z})$, in the cohomology spectral sequence $H^{p}\left(X, H^{q}(K(\mathbf{Z}, 2), \mathbf{Z})\right) \Rightarrow H^{*}\left(Y_{\delta}, \mathbf{Z}\right)$, we have only the single differential $d_{3}: E_{3}^{p, q} \rightarrow E_{3}^{p+3, q-2}$ sending $1 \otimes y \in E_{3}^{0,2}$ to $N x \otimes 1 \in E_{3}^{3,0}$ and thus sending $1 \otimes y^{n} \in E_{3}^{0,2 n}$ to $(N n) x \otimes y^{n-1} \in E_{3}^{3,2 n-2}$. From this one can compute the differential $d_{3}$ in the spectral sequence $H^{p}\left(X, R K^{1}(K(Z, 2))\right) \Rightarrow R K^{*}\left(Y_{\delta}\right)$. We do not need the full result, but anyway, from the commutative diagram

$$
\begin{gathered}
\mathbb{Z} \cong H^{0}\left(S^{3}, K^{3}(p t)\right) \stackrel{d_{3}}{\longrightarrow} \quad H^{3}\left(S^{3}, K^{0}(p t)\right) \cong \mathbf{Z} \\
\downarrow \\
\mathbb{Z}[[c]] \cong H^{0}\left(S^{3}, R K^{2}(K(\mathbf{Z}, 2))\right) \stackrel{d_{3}}{\longrightarrow} H^{3}\left(S^{3}, R K^{0}(K(\mathbf{Z}, 2))\right) \cong \mathbb{Z}[[c]]
\end{gathered}
$$


we can deduce that $d_{3}$ in the top spectral sequence is multiplication by $N$, that is, cup-product with $\delta$.

\section{The real case}

In this section we make a few remarks on real continuous-trace algebras, amplifying comments in [10]. A real $C^{*}$-algebra $A$ is a Banach *-algebra over $\mathbf{R}$ which is isometrically *-isomorphic to a norm-closed *-algebra of operators on a real Hilbert space. Giving such an $A$ is obviously equivalent to giving its complexification $A_{\mathrm{C}}$, together with the conjugation $\sigma$ of $A_{\mathrm{C}}$ of period 2. Alternatively, one may specify the map $a \mapsto \sigma(a)^{*}$, a complex-linear involutive *-preserving antiautomorphism of $A_{\mathrm{C}}$. Such involutions have been studied intensively in connection with the theory of Jordan algebras of operators.

We call a real $C^{*}$-algebra $A$ a real continuous-trace algebra if $A_{\mathbb{C}}$ is of continuous trace. Such an algebra has certain obvious invariants, namely the spectrum $X=\left(A_{\mathbf{C}}\right)^{\wedge}$, the involutive homeomorphism $\tau$ induced on $X$ by $\sigma$, and the Dixmier-Douady class $\delta \in H^{3}(X, Z)$. The following fact was pointed out to me by Phil Green many years ago.

Proposition 3.1. With notation as above, $\tau^{*}(\delta)=-\delta$.

Proof. This follows from the fact (due to Phil Green-see [12] for a detailed exposition) that if $B$ is any complex continuous-trace algebra, then $\delta\left(B^{\text {op }}\right)=-\delta(B)$, where $B^{\text {op }}$ is $B$ with multiplication reversed. We apply this to $B=A_{\mathrm{C}}$. The conjugation $\sigma$ induces an isomorphism $B \rightarrow B^{\text {op }}$ which on $\hat{B}=X$ is given by $\tau$, and thus $\tau^{*}\left(\delta\left(B^{\text {op }}\right)\right)=\delta(B)$, that is $\tau^{*}(-\delta)=\delta$.

Corollary 3.2. Suppose $\delta \in H^{3}(X, Z)$ and $\delta$ is not conjugate to $-\delta$ under a homeomorphism of $X$.Then the complex stable continuous-trace algebra $A_{\delta}$ has no real structure. In particular this applies if $X$ is an oriented 3-manifold with no orientation-reversing homeomorphism, and $\delta \neq 0$ in $H^{3}(X, Z) \cong Z$.

Now let $\mathscr{H}_{R}, \mathscr{H}_{C}$, and $\mathscr{H}_{H}$ be separable infinite-dimensional real, complex, and quaternionic Hilbert spaces, respectively. Let $\mathscr{K}\left(\mathscr{K}_{R}\right)=\mathscr{K}_{R}, \mathscr{K}\left(\mathscr{H}_{C}\right)=$ $\mathscr{K}$, and $\mathscr{K}\left(\mathscr{K}_{H}\right)=\mathscr{K}_{H}$ be the corresponding algebras of compact operators. We call $A$ stable if $A \cong A \otimes \mathscr{K}_{\mathrm{R}}$; here $\otimes$ denotes of course the tensor product of real $C^{*}$-algebras. Observe that since $\mathscr{H}_{C} \cong \mathscr{H}_{R} \otimes_{R} C$ and $\mathscr{H}_{\mathbf{H}} \cong \mathscr{H}_{R} \otimes_{R} H$, we have $\mathscr{K} \cong \mathscr{K}_{R} \otimes C, \mathscr{K}_{H} \cong \mathscr{K}_{R} \otimes H$, so all three of $\mathscr{K}_{R}, \mathscr{K}$, and $\mathscr{K}_{H}$ are stable. These are, in a sense to be made precise shortly, the prototypes for all stable separable real continuous-trace algebras. 
Now consider a stable separable real continuous trace algebra $A$. Then $A_{\mathrm{C}}$ is stable in the sense of complex algebras, so $A_{\mathrm{C}} \cong A_{\delta}$ for $\delta \in H^{3}(X, \mathrm{Z})$ as above. The involutive homeomorphism $\tau$ of $X$ defines a closed subset $X^{\tau}$ which as we explained in [10] splits into a disjoint union of two closed subsets $X_{\mathbf{R}}$ and $X_{\mathbf{H}}$. These are defined by the property that if $x \in X^{\tau}$, so that $x$ defines an irreducible complex representation of $A_{\mathrm{C}}$ which is the complexification of an irreducible representation of $A$, then in one case the image of $A$ is isomorphic to $\mathscr{K}_{R}$ and in the other case it is isomorphic to $\mathscr{K}_{H}$. (This makes sense since $\mathscr{K}_{\mathrm{A}}$ and $\mathscr{K}_{\mathrm{H}}$ both have complexification $\mathscr{K}$, the latter since $\left(\mathscr{K}_{\mathbf{H}}\right)_{\mathbf{C}} \cong\left(\mathscr{K}_{\mathbf{R}} \otimes \mathbf{H}\right)_{\mathbf{C}} \cong \mathscr{K} \otimes_{\mathbf{C}}\left(\mathbf{H} \otimes_{\mathbf{R}} \mathbf{C}\right) \cong \mathscr{K} \otimes_{\mathbf{C}} M_{2} \cong M_{2}(\mathscr{K}) \cong \mathscr{K}$.) Furthermore $\tau$ acts freely on $X-X^{\tau}$. Thus $A$ splits as an extension

$$
0 \rightarrow A_{1} \rightarrow A \rightarrow A_{2} \oplus A_{3} \rightarrow 0
$$

where $A_{1}$ is of complex type and $A_{2}$ and $A_{3}$ are of real and quaternionic types, respectively. The notion of type may be formally defined by the property that $A$ is of real (respectively, complex, quaternionic) type if each irreducible representation of $A$ (on a real Hilbert space) has image isomorphic to $\mathscr{K}_{\mathrm{R}}$ (respectively, $\mathscr{K}, \mathscr{K}_{H}$ ). While the extension $(*)$ may presumably be relatively complicated to describe, we shall at least give a classification of the algebras of pure type. The letter $w$ is used in the theorem since it basically stands for a Stiefel-Whitney class.

THEOREM 3.3. Let $A$ be a stable separable real continuous-trace algebra of real (respectively quaternionic) type, with $\left(A_{\mathrm{C}}\right)^{\wedge}=X$. Then $A \cong \Gamma_{0}(\mathscr{A})$, where $\mathscr{A}$ is a locally trivial bundle of real $C^{*}$-algebras over $X$, with fibres isomorphic to $\mathscr{H}_{R}$ (respectively $\mathscr{K}_{H}$ ). The bundle $\mathscr{A} \rightarrow X$ is determined by a characteristic class $w \in H^{2}\left(X, Z_{2}\right)$, and this determines $A$ up to spectrum-fixing isomorphism. The Dixmier and Douady invariant of $A_{\mathrm{C}}$ is given by $\beta(w)$, where $\beta: H^{2}\left(X, Z_{2}\right) \rightarrow H^{3}(X, Z)$ is the Bockstein homomorphism (that is, connecting homomorphism in the long exact cohomology sequence associated to the short exact sequence $0 \rightarrow \mathbf{Z} \stackrel{2}{\rightarrow} \mathbf{Z} \rightarrow \mathbf{Z}_{2} \rightarrow 0$ of constant sheaves).

Conversely, given $w \in H^{2}\left(X, \mathbf{Z}_{2}\right)$, there exist unique corresponding stable separable real continuous-trace algebras $A_{w}^{\mathbf{R}}$ and $A_{w}^{\mathbf{H}}$ of real and quaternionic type, respectively, giving rise to the characteristic class $w$. These are related by $A_{w}^{\mathbf{H}} \cong A_{w}^{\mathbf{R}} \otimes \mathbf{H}, A_{w}^{\mathbf{R}} \cong A_{w}^{\mathbf{H}} \otimes \mathbf{H}$.

Finally, $\delta \in H^{3}(X, Z)$ arises as the Dixmier-Douady class of $A_{\mathrm{C}}$, for some stable separable real continuous-trace algebra $A$ of real or quaternionic type, if and only if $2 \delta=0$. When this holds, the possible $A$ 's of the same type may be parameterised (up to spectrum-fixing isomorphism) by $\beta^{-1}(\delta) \subseteq H^{2}\left(X, \mathbf{Z}_{2}\right)$.

Proof. The local triviality may be proved by exactly the same method used in [5] in the complex case. Alternatively, we may begin with the result 
of Theorem 1.1 that $A_{\mathrm{C}} \cong \Gamma_{0}\left(\mathscr{A}_{\mathrm{C}}\right)$, where $\mathscr{A}_{\mathrm{C}}$ is a locally trivial bundle of complex $C^{*}$-algebras over $X$, with fibres $\cong \mathscr{K}$. The conjugation $\sigma$, being an isometric *-automorphism of $A_{\mathrm{C}}$, must then be given by a continuous section of the bundle of conjugations of the fibres of $A_{\mathrm{C}}$ of real (respectively, quaternionic) type. Now note that if we fix an identification of $\mathscr{H}_{C}$ with $\mathscr{H}_{R} \otimes_{\mathbf{R}} \mathbb{C}$, and thus a "standard" conjugation - of real type, then any other conjugation of $\mathscr{K}$ must be of the form $\sigma: x \mapsto u \bar{x} u^{*}$, where $u \in \mathscr{U}$ is determined up to a scalar and $u \bar{u}=\gamma \in \mathbb{C}$ in order to guarantee that $\sigma^{2}=$ id. This means, since $u^{-1}=u^{*}=\bar{u}^{t}$ ( $t$ the transpose) that $u=\bar{\gamma} u^{t}$, hence, since ${ }^{t}$ has only the eigenvalues \pm 1 , that $\gamma=1$ (real case) or $\gamma=-1$ (quaternionic case). Thus the real conjugations correspond to symmetric unitaries modulo scalars and the quaternionic conjugations correspond to skew-symmetric unitaries modulo scalars. Now we can prove that $A \cong \Gamma_{0}(\mathscr{A})$ with $\mathscr{A}$ a locally trivial bundle of the sort described in the theorem. Since $\mathscr{A}_{C}$ is locally trivial, it is no loss of generality to assume $A_{\mathrm{C}} \cong C_{0}(X, \mathscr{K})$. Then by what we have seen, $\sigma$ is given by a continuous map

$$
f: X \rightarrow\left\{\dot{u} \in P \mathscr{U} \mid u^{t}= \pm u\right\},
$$

and

$$
A=\left\{\phi \in C_{0}(X, \mathscr{K}) \mid \phi(x)=f(x) \cdot \overline{\phi(x)} \text { for all } x \in X\right\} .
$$

If (in the real case) $f$ can be factored as $g g^{t}$, where $g: X \rightarrow P \mathscr{U}$ is continuous, then $\phi \mapsto g \cdot \phi$ gives a spectrum-preserving isomorphism from $C_{0}\left(X, \mathscr{H}_{\mathbf{R}}\right)$ to $A$. Now if $f$ lifts to a map $X \rightarrow\left\{u \in \mathscr{U} \mid u^{t}=u\right\}$, then $f$ has such a factorization (by simple spectral theory), and as $f$ always has such liftings locally (by local triviality of the projection $\mathscr{U} \rightarrow P \mathscr{U}$ ), we deduce that $A$ comes from a locally trivial field of elementary real $C^{*}$-algebras. The quaternionic case is similar.

Thus we have proved the first part of the theorem, and the rest reduces to bundle theory. The automorphism group of $\mathscr{K}_{\mathbf{R}}$ is $P \mathscr{O}=\mathscr{O} /\{ \pm 1\}$, where $\theta$ is the orthogonal group of $\mathscr{H}_{R}$ with the weak operator topology, and $\{ \pm 1\}$ is of course the centre of $\mathscr{\theta}$. As in the complex case, $\mathscr{\theta}$ is contractible, so $\operatorname{Aut}\left(\mathscr{K}_{R}\right)$ is a classifying space for $Z_{2}$, that is, a $K\left(Z_{2}, 1\right)$-space. A similar analysis applies in the quaternionic case, with $\mathscr{O}$ replaced by $\mathscr{S}_{\mu}$ (the infinite-dimensional symplectic group). Thus in both the real and quaternionic cases, $\mathscr{A}$ comes from a principal bundle $Y$ over $X$ whose fibres are topological groups with the homotopy type of a $K\left(Z_{2}, 1\right)$. Thus the possibilities for $Y$ are given by $\left[X, K\left(Z_{2}, 2\right)\right]=H^{2}\left(X, Z_{2}\right)$. It is also clear that any $w \in H^{2}\left(X, \mathbf{Z}_{2}\right)$ defines a principal bundle $Y_{w}$ and then bundles of algebras with fibres isomorphic to $\mathscr{K}_{\mathrm{R}}$ or $\mathscr{K}_{\mathrm{H}}$. The sections of these bundles which vanish at infinity then give the algebras $A_{w}^{R}$ and $A_{w}^{\mathbf{H}}$. The relation between these comes from the familiar isomorphisms

$$
\mathscr{K}_{R} \otimes H \cong \mathscr{K}_{H}, \quad \mathscr{K}_{H} \otimes H \cong \mathscr{K}_{R} \otimes\left(H \otimes_{R} H\right) \cong \mathscr{K}_{R} \otimes M_{4}(\mathbf{R}) \cong \mathscr{K}_{R} .
$$


It remains to prove the statement about $\delta=\delta\left(A_{\mathrm{C}}\right)$. Given $Y_{w}$, realised say as a bundle with fibres $P \mathscr{O}$, the bundle $Y_{\delta}$ with fibres $P \mathscr{U}$ is obviously the associated bundle coming from the inclusion $P \mathscr{O} \rightarrow P \mathscr{U}$. From the homotopy point of view this is just the natural map of classifying spaces $B Z_{2} \rightarrow B T$. But from the short exact sequence of abelian groups $1 \rightarrow Z_{2} \rightarrow T \stackrel{2}{\rightarrow} \mathrm{T} \rightarrow 1$ we obtain a fibration $B Z_{2} \rightarrow B T \rightarrow B T$, and hence the Bockstein exact sequence

$$
\cdots \rightarrow[X, K(\mathbb{Z}, 2)] \stackrel{2}{\rightarrow}[X, K(\mathbf{Z}, 2)] \rightarrow\left[X, K\left(\mathbf{Z}_{2}, 2\right)\right] \stackrel{\beta}{\rightarrow}[X, K(\mathbf{Z}, 3)] \rightarrow \cdots .
$$

So a principal $P \mathscr{U}$-bundle with Dixmier-Douady class $\delta$ has a reduction to a principal $P \mathscr{O}$-bundle if and only if $2 \delta=0$. In this case, from the exact sequence, the possible reductions are given by $\beta^{-1}(\delta)$. The quaternionic case is homotopy-theoretically the same, so this completes the proof.

Note also that all the comments we made about automorphisms and $K$ theory of $A_{\delta}$ have obvious analogues in the real and quaternionic cases, with the proofs the same as in Sections 1 and 2 . We merely state the results.

THEOREM 3.4. Let $X$ be a second-countable locally compact space, let $w \in$ $H^{2}\left(X, \mathbf{Z}_{2}\right)$, and let $A_{w}$ denote either $A_{w}^{\mathbf{R}}$ or $A_{w}^{\mathbf{H}}$. There are short exact sequences of groups:

(a) $1 \rightarrow$ Aut $_{X} A_{w} \rightarrow$ Aut $A_{w} \rightarrow$ Homeo $_{w} X \rightarrow 1$,

(b) $1 \rightarrow \operatorname{Inn} A_{w} \rightarrow$ Aut $_{X} A_{w} \rightarrow H^{1}\left(X, \mathbf{Z}_{2}\right) \rightarrow 1$.

THEOREM 3.5. Let $X, w$ be as above and define $K O^{-i}(X, w)=K O_{i}\left(A_{w}^{\mathbf{R}}\right)$. (One can also define $K \mathrm{Sp}^{-i}(X, w)$ using $A_{w}^{\mathrm{H}}$, but these are the same groups with a degree shift of 4, by periodicity of the real Clifford algebras.) If $X$ is a finite $\mathrm{CW}$-complex, there is a commuting diagram of Atiyah-Hirzebruch-type spectral sequences

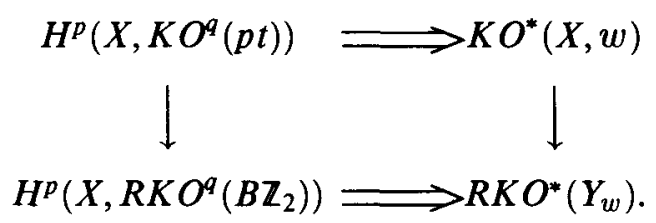

Here the groups $R K O^{q}\left(B Z_{2}\right)$ are computed in [3].

We proceed now to the study of algebras of complex type, which is slightly harder because of the presence of the involution $\tau$. This obviously is a necessary ingredient in the classification.

THEOREM 3.6. Let $A$ be a stable separable real continuous-trace algebra of complex type, with $\left(A_{\mathbb{C}}\right)^{\wedge}=X$ and induced involution $\tau$. Let $\bar{X}=X / \tau$. Then $A \cong \Gamma_{0}(\mathscr{A})$, where $\mathscr{A}$ is a locally trivial bundle of real $C^{*}$-algebras 
over $\bar{X}$, with fibres isomorphic to $\mathscr{K}$. The structure group of the bundle $\mathscr{A}$ is $P \mathscr{U}^{\prime}=\mathscr{U}^{\prime} / \mathrm{T}$, where $\mathscr{U}^{\prime}$ is the group of unitary or conjugate-unitary operators on $\mathscr{X}_{\mathrm{C}}$, with the weak operator topology. There are group extensions

$$
1 \rightarrow \mathscr{U} \rightarrow \mathscr{U}^{\prime} \rightarrow \mathbf{Z}_{2} \rightarrow 1, \quad 1 \rightarrow P \mathscr{U} \rightarrow P \mathscr{U}^{\prime} \rightarrow \mathbf{Z}_{2} \rightarrow 1,
$$

where $\mathbf{Z}_{2}$ is the cyclic group of two elements, identified with $\mathrm{Gal}(\mathbf{C} / \mathbf{R})$.

Thus $\mathscr{A}$ is the $\mathscr{K}$-bundle associated to a principal $P \mathscr{U}^{\prime}$-bundle $Y \rightarrow \bar{X}$. The classifying element of this bundle in $H^{1}\left(\bar{X}, \underline{P \mathscr{U}^{\prime}}\right)=\left[\bar{X}, B P \mathscr{U}^{\prime}\right]$ maps to the classifying element of the two-fold covering $p: X \rightarrow \bar{X}$ defined by $\tau$ in $H^{1}\left(\bar{X}, \mathbf{Z}_{2}\right) \cong\left[\bar{X}, B Z_{2}\right]$. Conversely, to any element $\phi \in\left[\bar{X}, B P \mathscr{U}^{\prime}\right]$ mapping to the classifying element for $\tau$ in $H^{1}\left(\bar{X}, \mathbf{Z}_{2}\right)$ is a stable separable real continuoustrace algebra $A_{\phi}$ of complex type with $\left(\left(A_{\phi}\right)_{\mathrm{C}}\right)^{-} \cong X$ and $\tau$ as given. $A_{\phi}$ is unique up to spectrum-fxing *-isomorphisms. The Dixmier-Douady class of $\left(A_{\phi}\right)_{\mathrm{C}}$ is the homotopy class $\delta \in[X, B P \mathscr{U}] \cong H^{3}(X, Z)$ defined by the pull-back diagram

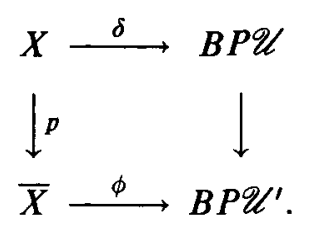

(Note that $B P \mathscr{U}$, a $K(\mathbf{Z}, 3)$-space, is a double cover of $B P \mathscr{U}^{\prime}$.)

Proof. Once again, there are two ways of proving the local triviality. One is by imitating the method of [5]. We give the other method, which is to make use of the fact that $A_{\mathrm{C}} \cong \Gamma_{0}\left(X, \mathscr{A}_{\mathrm{C}}\right)$, where $\mathscr{A}_{\mathrm{C}}$ is a locally trivial bundle of elementary complex $C^{*}$-algebras. Now in the complex case, each irreducible quotient of $A$ is isomorphic to $\mathscr{K}$, which has complexification $\cong \mathscr{K} \oplus \mathscr{K}$. So $\tau$ acts freely and $p: X \rightarrow \bar{X}$ is a two-to-one covering. Since $p$ is locally trivial, to prove local triviality of $A$ we may without loss of generality replace $X$ by an open set over which $p$ is trivial, and assume $X=\bar{X} \amalg \bar{X}$ with $\tau$ interchanging the two copies. Then $\delta$, the Dixmier-Douady invariant of $A_{\mathrm{C}}$, is of the form $\left(\delta_{1},-\delta_{1}\right), \delta_{1} \in H^{3}(\bar{X}, \mathbf{Z})$, so $A_{\mathrm{C}} \cong A_{\delta_{1}} \oplus A_{-\delta_{1}}$. Then $A$ is the fixed point algebra of $\sigma$, and we claim projection onto the first factor of $A_{\delta_{1}}$ gives a real linear ( $A$ does not have a natural complex structure) *isomorphism $A \rightarrow A_{\delta_{1}}$. Indeed, this is immediate since complexifying gives a *-homomorphism $A_{\mathrm{C}} \rightarrow\left(A_{\delta_{1}}\right) \mathrm{C}$ which is injective and locally surjective, hence an isomorphism. Thus $A \cong \Gamma_{0}(\mathscr{A})$, with $\mathscr{A}$ a locally trivial bundle over $\bar{X}$ with fibres $\cong \mathscr{H}$.

Next, observe that the structure group of the bundle $\mathscr{A}$ is the group of *-automorphisms of $\mathscr{K}$ viewed as a real algebra. Any such *-automorphism induces a real-linear automorphism of the centre $\mathbb{C}$ of the multiplier algebra, 
that is, an element of the Galois group $\mathrm{Gal}(\mathbb{C} / \mathbf{R})$. If this Galois element is trivial, the automorphism is complex-linear, hence of the form Ad $u$ for some $u \in \mathscr{U}$. Otherwise it is conjugate-linear and must come from a conjugateunitary $u \in \mathscr{U}^{\prime}$. (The $u$ can be recovered up to scalars from the action of the automorphism on rank-one projections.) So this proves the second statement.

Finally, we must prove the statement about classification. The bundle $\mathscr{A}$ must arise from a principal $P \mathscr{U}^{\prime}$-bundle over $\bar{X}$, and since we need compatibility with the principal $P \mathscr{U}$-bundle defining $\mathscr{A}_{\mathrm{C}}$ over $X$, everything else follows.

Note that given $\phi \in\left[\bar{X}, B P \mathscr{U}^{\prime}\right]$ compatible with the covering $p: X \rightarrow \bar{X}$ defined by $\tau: X \rightarrow X$ with $\bar{X}=X / \tau$, we can once again define twisted $K$-groups $K R^{-i}(X, \tau, \phi)=K O_{i}\left(A_{\phi}\right)$. In fact, given $\tau$, there is always a "trivial" choice for $\phi$, with $A_{\phi}=C_{0}(X, \tau) \otimes \mathscr{K}$, where as in [16, Proposition 2.2], $C_{0}(X, \tau)=\left\{f \in C_{0}(X) \mid \bar{f}=f \circ \tau\right\}$. This shows that the fibration $B P \mathscr{U} \rightarrow B P \mathscr{U}^{\prime} \rightarrow B Z_{2}$ splits, and is really just a twisted product $K(\mathbf{Z}, 3) \rtimes K\left(\mathbf{Z}_{2}, 1\right)$, where the generator of $\pi_{1}\left(K\left(\mathbf{Z}_{2}, 1\right)\right)$ acts by -1 on $\pi_{3}(K(\mathbf{Z}, 3))$. Thus to describe $\phi$, it is equivalent to give a class in $H^{3}(\bar{X}, \mathscr{Z})$, where $\mathscr{Z}$ is a sheaf on $\bar{X}$ locally isomorphic to $Z$. This may be viewed as a twisted Dixmier and Douady class.

\section{References}

[1] J. F. Adams, Stable Homotopy and Generalised Homology, (Chicago Lectures in Math., Univ. of Chicago Press, Chicago and London, 1974).

[2] C. A. Akemann, G. K. Pedersen and J. Tomiyama, 'Multipliers of $C^{*}$-algebras', J. Funct. Anal. 13 (1973), 277-301.

[3] M. F. Atiyah and G. Segal, 'Equivariant $K$-theory and completion,' J. Differential Geom. 3 (1969), 1-18.

[4] J. Dixmier, $C^{*}$-algebras, (North-Holland, Amsterdam and New York, 1977).

[5] J. Dixmier and A. Douady, 'Champs continus d'espaces Hilbertiens et de $C^{*}$-algèbres,' Bull. Soc. Math. France 91 (1963), 227-284.

[6] P. Donovan and M. Karoubi, 'Graded Brauer groups and $K$-theory with local coefficients', Publ. Math. I.H.É.S. 38 (1970), 5-25.

[7] A. Grothendieck, Le Groupe de Brauer, I: Algèbres d'Azumaya et Interprétations Diverses, Séminaire Bourbaki, Paris, $17^{\mathrm{e}}$ année (1964/64), exposé 290.

[8] M. Karoubi, K-Theory: An Introduction, (Springer, Berlin, Heidelberg, and New York, 1978).

[9] N. H. Kuiper, 'The homotopy type of the unitary group of Hilbert space', Topology 3 (1965), 19-30.

[10] I. Madsen and J. Rosenberg, 'The universal coefficient theorem for equivariant $K$-theory of real and complex $C^{*}$-algebras', Index Theory of Elliptic Operators, Foliations, and Operator Algebras, edited by J. Kaminker, K. Millett, and C. Schochet, (Contemp. Math., vol. 70, Amer. Math. Soc., Providence, R.I., 1988, pp. 145-173). 
[11] J. A. Mingo, K-Theory and Multipliers of Stable $C^{*}$-Algebras, (Ph.D thesis, Dalhousie Univ., Halifax, N.S., 1982).

[12] E. M. Parker, 'The Brauer group of graded continuous-trace $C^{*}$-algebras', preprint, 1987.

[13] J. Phillips and I. Raeburn, 'Automorphisms of $C^{*}$-algebras and second Čech cohomology', Indiana Univ. Math. J. 29 (1980), 799-822.

[14] J. Rosenberg, 'Homological invariants of extensions of $C^{*}$-algebras', Operator Algebras and Applications, edited by R.V. Kadison, (Proc. Sympos. Pure Math., vol. 38, Part 1, Amer. Math. Soc., Providence, R.I., 1982, pp. 35-75).

[15] J. Rosenberg, 'The role of $K$-theory in non-commutative algebraic topology', Operator Algebras and $K$-Theory, edited by R. G. Douglas and C. Schochet, (Contemp. Math., vol. 10, Amer. Math. Soc., Providence, R.I., 1982, pp. 155-182).

[16] J. Rosenberg, ' $C$ *-algebras, positive scalar curvature, and the Novikov conjecture, III,' Topology 25 (1986), 319-336.

[17] G. Segal, 'Classifying spaces and spectral sequences', Publ. Math. I.H.E.S. 34 (1968), 105112.

Department of Mathematics

University of Maryland

College Park, Maryland 20742

U.S.A. 